\title{
Distribution Network Reconfiguration Based on Niching Techniques Particle Swarm Optimization Algorithm
}

\author{
XuAoran Zhang Liu Gao Yang Gu Cailian Qu Chunyu \\ Shenyang Institute of Engineering ShenyangNo.18 Puchang Road Shenbei District, \\ Shenyang, Liaoning, P.R.C.
}

Xar1868@163.com

Key words: Distribution Network;Network Reconfiguration;Niching Techniques;Particle Swarm Optimization;

Abstract: Distribution network reconfiguration is an important tool to optimize the operating conditions of a distribution network. Niche Binary Particle Swarm Optimization (NBPSO) Algorithm is proposed to overcome the defect of PSO prematurity for distribution network reconfiguration. In view of distribution network switch location in order, the weighted hamming distance is redefined for dividing different niche groups. Different niche groups are optimized using PSO, and particles in renewal groups are adapted by sharing mechanism. An IEEE 33-bus radial distribution test system is taken as a study system for performing the test. The results reveal the strong global searching of the proposed method for solving the problem.

\section{Introduction}

Distribution network with closed loop design, the characteristics of the open-loop operation ${ }^{[2]}$, contains a lot of disconnect switches and contact switches.Distribution network reconfiguration is adjusted to the closure of the switch optimization distribution network operation, in order to reduce the loss, load balance, eliminate overload and improve power supply quality and economical operation of the system requirements ${ }^{[1]}$.Distribution network reconfiguration is a highly non-linear combination of the optimization problem is NP-hard problem in the mathematical sense.

\section{Niche particle swarm algorithm}

The algorithm is a social group behavior-based bionic global optimization algorithm, with memory features.The basic idea is that each particle represents a potential solution of optimization problem, the optimization function determines the appropriate value of each particle, by real-time tracking of populations of the global optimal value and individual current optimal value to adjust the positions and velocities of all particles, finally get the optimal solution of optimization problems.

$$
\begin{aligned}
V_{i d}^{k+1} & =\omega V_{i d}^{k}+c_{1} \mathrm{r}_{1}\left(P_{i d}^{k}-x_{i d}^{k}\right)+c_{2} \mathrm{r}_{2}\left(P_{g d}^{k}-x_{i d}^{k}\right) \\
x_{i d}^{k+1} & =x_{i d}^{k}+V_{i d}^{k+1}
\end{aligned}
$$


Type of: $\mathrm{x}, \mathrm{V}$, respectively, for the position and velocity of the particles, $\mathrm{k}$ is the particle number of iterations, $\omega_{\text {is }}$ the inertia weight, c1, c2, a non-negative constant, called the learning factor, $\mathrm{r} 1$ and $\mathrm{r} 2,[0,1] \mathrm{random}$ number within the interval, $P_{\mathrm{id}}^{\mathrm{k}}, P_{\mathrm{gd}}^{\mathrm{k}}$, respectively, corresponding with the individual optimal value of the particles and all particles of the optimal value of the position.

The basic binary particle swarm into discrete, particle position of each dimension state is limited to 0 and $1^{[3]}$, the speed of iterative formula and the basic particle swarm algorithm, but the speed in a certain sense becomes the position of the particle is close to the 1or0 probability of decision factors, if the particle velocity of $V_{\mathrm{idd}}^{k+1}, x_{\mathrm{id}}^{k+1}$ positionlthe greater probability, and vice versa. The Sigmoid function will be responsible for the speed $V_{\mathrm{id}}^{\mathrm{k}+1}$ into $[0,1]$ between the numerically, the formula is:

$S\left(V_{i d}^{k+1}\right)=\frac{1}{\exp \left(-V_{i d}^{k+1}\right)}$

The location of the iteration formula is as follows:

$x_{i d}^{k+1}=\left\{\begin{array}{l}1, \operatorname{rand}()<S\left(V_{i d}^{k+1}\right) \\ 0, \text { other }\end{array}\right.$

The rand $[0,1]$ within the interval of random number.

Niche technique ${ }^{[4]}$ to achieve there are basically four types based on the pre-selection mechanism, based on the crowding out mechanism, based on the total fitness function and clustering-based niche technology.Niche technology with microscopic and scalpel type micro local optimization effect, after the study of the technology and particle swarm optimization PSO algorithm, to a certain extent made up of particle swarm algorithm local search ability insufficient characteristic.

\section{Algorithm in Power Distribution System Reconfiguration}

Distribution network reconfiguration problem is actually a series of switches to open the closed state of the orderly combination of problems. In a mathematical sense, can be 0,1 , the opening and closing of the switch status, the corresponding particle swarm algorithm for particle position on the various dimensions of State.Neither the network nor of the combined distribution network topological constraints of island, in order to improve computational efficiency, some special switch code below accordingly: (1) and connected to the power switch power supply if broken will cause you to lose, never reset the closed state, encoding can not be considered;(2) distribution tree branch did not form a ring in switch, if broken will form an island, so this type of switch you can always reset the closed state, when encoding is 
not taken into account.After processing, the particle location of optimized dimension can also be reduced, improving the efficiency optimization.

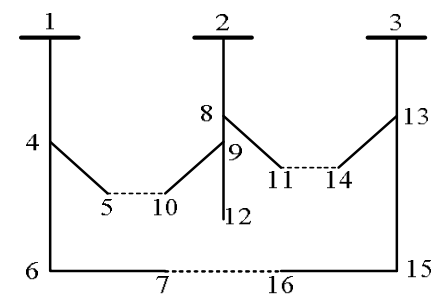

Figure 1 feeder figure 16 nodes

At the same time in order to more effectively infeasible solution of distinguishing, the encoding based on the basic loop [10].IEEE16 nodes, for example, node 1-4-5-10-8-9-2 composed of loops and nexus 8-1-14-13-3 make up the round, three nodes composed of 4-6-7-16-15-13 loops.After taking into account the encoding to simplify, to encode three loops in order, as shown in Figure loop code for loop-loop-loop three-three of the second power supply branch is not taken into account, there is code between 9-12 not taken into account.

\section{example analysis}

Branch-1 connected to the power of the network, in accordance with simplification of coding methods, you can optimize resets when closed, not taken into account, so each particle has $36 \mathrm{~d}$, taking population size of $30, \omega$ for $0.9, \mathrm{C} 1, \mathrm{C} 2$ learning factor is taken as 2 , maximum number of iterations is 150 times.After using NBPSO algorithm for reconfiguration of the results.

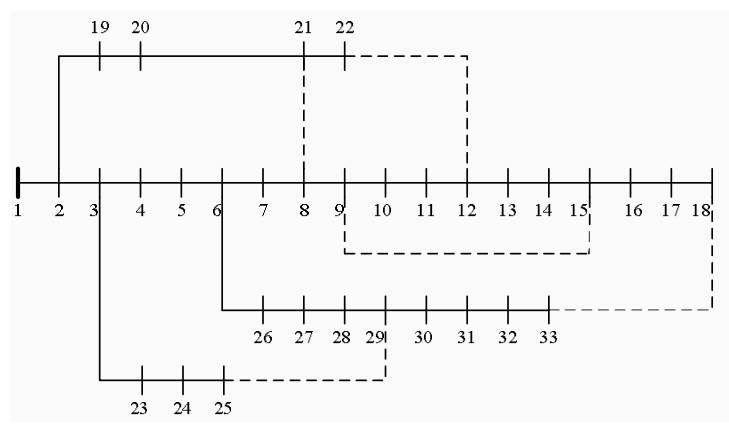

Fig.2 IEEE33 notes system network in figure

Standard test system IEEE33 node node voltage after remodeling trend chart as shown in Figure 3.

In order to analyze the niche binary Particle Swarm Optimization performance, reconstruction of 20 tests, where the minimum number of iterations is 4 times, maximum number of iterations is 32 times, average number of iterations is 13.6 times, reconstruction of 20 test is 20 times the number in the optimal solution. These results indicate that this reconstruction algorithms to effectively increase the efficiency of search optimization.

\section{conclusion}

This will small Habitat II into system particle group algorithm application to solution 
distribution grid heavy frame problem Shang, combination distribution grid heavy frame of features [8], simplified has coding, again defined has Division niche border of standard Hamming distance, by population initial of and update in the on not line solutions of repair, avoid has eventually not line solutions of produced, overcome has single particle group algorithm of early problem.IEEE33 node typical testing system for the calculation, simulation results indicate that the algorithm to reduce losses, improve search efficiency.

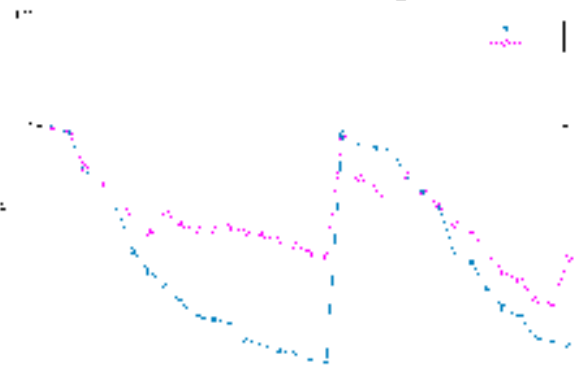

Fig.3 Trend Graph of IEEE33 notes system network

\section{Acknowledgements}

The research work was supported by the department of Liaoning education scientific research project.

\section{Reference}

[1] XU Bing-yin, LI Tian-you, XUE Yong-duan. Smart Distribution Grid and Distribution Automation[J]. Automation of Electric Power Systems, 2009, 33(17): 38-41.

[2] LIU Li,CHEN Xue-yun.Reconfiguration of distribution networks based on fuzzy genetic algorithm[J]. Proceedings of the CSEE,2000,20(2): 66-69.

[3] LU Zhi-gang, YANG Guo-liang,ZHANG Xiao-hui,et al.Reconfiguration of distribution network based onimproved particle swarm optimization[J]. Power System Protection and Control,2009,37(7): 30-34.

[4]WANG Chun,CHENG Hao-zhong.Reconfiguration of distribution network based on plant growth simulation algorithm[J]. Proceedings of the CSEE,2007,27(19) : 50-55.

[5] Kennedy J,Barnhart R.Particle swarnl optimization[C].Proceeding of the IEEE International Conference on Neural Networks,IEEE Neural Networks Council,1995: 1942-1948. 